\title{
Review
}

\section{Research Progress on the Relationship Between Oral Microbial Community and Tumor}

\author{
Shujun Ma \\ Department of Medical Oncology, Shandong University Affiliated Shandong Provincial Hospital, Jinan China
}

\section{Keywords \\ Oral microbial community; Oral squamous cell carcinoma; Human immunodeficiency virus; Helicobacter pylori}

\section{Correspondence}

Shujun Ma,

E-mail: sjmajn@163.com

DOI: $10.1515 / i i-2017-0123$

\begin{abstract}
Significant progress was observed in studies of the relationship between oral Helicobacter pylori and gastric cancer and tumors. Based on three distinct and close relationships, namely, the relationship between oral $H$. pylori and gastric cancer, between oral microbial communities and oral squamous cell carcinoma, and between oral microbial communities of human immunodeficiency virus-infected patients and tumors, this work reviews the relationship between oral microbial communities and tumors. This research also provides reference for further analysis of the relationship between oral microorganisms and tumors to realize early diagnosis of tumor patients through detecting oral microorganisms under adjuvant therapy.
\end{abstract}

Microorganisms residing in the mouth include bacteria, fungi, and viruses, with bacteria representing dominant communities. Microorganisms compose one of the most complex microbial communities ${ }^{[1]}$. By the end of 19 th century, researchers discovered that microbes cause some serious diseases. By controlling infection and transmission of microorganisms, significant progress was achieved in treatment of some diseases. Literature ${ }^{[2]}$ proved that $20 \%$ of cancers are associated with microbial infection. For example, Helicobacter pylori is closely associated with occurrence of gastric adenocarcinoma and mucosaassociated lymphoid tissue lymphoma; Epstein-Barr virus is associated with Burkitt lymphoma and nasopharyngeal carcinoma. These studies suggested that a close relationship may exist between oral microbial communities and tumors. To date, a large number of studies explored the relationship between oral microbial infection and tumor development. These investigations were limited to descriptive studies and possible mechanisms but provided possible direction to further strengthen studies of mechanism and to realize prevention and treatment of clinical tumors. This work mainly introduces three views. First, H. pylori and gastritis and gastric cancer are related. Mouth is located at the front of digestive tract. The relationship between $H$. pylori infection in the mouth and gastritis and gastric cancer attracted considerable attention. Second, oral squamous carcinoma is the most common oral tumor. This research mainly focuses on relationship of oral squamous carcinoma with microbial community. Third, oral microbial communities of human immunodeficiency virus (HIV)-infected patient show specificity. Thus, HIV patients display high risks of suffering from tumors. Scholars constantly paid attention to the relationship between oral microbial communities and tumors.

\section{Relationship between oral H. pylori and gastric cancer}

Relationship between oral $H$. pylori and $H$. pylori infection in the stomach

H. pylori is a Gram-negative, aerobic screw bacterium. Main routes of $H$. pylori transmission include fecal-oral, oral-oral, and gastric-oral routes ${ }^{[3]}$. Thus, oral health status directly or indirectly affects $H$. pylori infection and reinfection of the stomach. H. pylori is found in saliva, biomembrane on back of the tongue, dental plaques, oral ulcers, and oral cancers. Majmudar et al. ${ }^{[4]}$ separated H. pylori from dental plaques of 40 healthy individuals. $H$. pylori that commonly exists in 
the mouth is associated with gastric colonization. Clinical research ${ }^{[5]}$ showed that $H$. pylori can be detected from the mouth of $97 \%$ of volunteers, whereas $26.2 \%$ of volunteers suffered from $H$. pylori infection gastritis. Miyabayashi et al. [6] observed that effects of systemic antibacterial therapy on $H$. pylori infection gastritis is associated with colonization of $H$. pylori infection in the mouth. Oral cavity is an important reservoir of $H$. pylori and significantly influences $H$. pylori infection in the stomach.

\section{Relationship between $H$. pylori and gastritis and gastric cancer}

H. pylori is related to chronic antral gastritis and peptic ulcer and is clearly related to gastric cancer ${ }^{[7]}$. In 1994, the World Health Organization considered H. pylori as a class I carcinogen based on epidemiological investigations. $H$. pylori can directly regulate function of gastric epithelial cells through its virulence factors, and it can also cause inflammation, indirectly affecting gastric epithelial cells $[8,9]$. These two processes commonly promote occurrence and development of gastric cancer. In conclusion, $H$. pylori in the oral cavity plays an important role in occurrence and development of gastric cancer.

\section{Relationship between oral microorganisms and oral squamous carcinoma}

Oral squamous carcinoma is the most common malignant tumor in the oral cavity. Prevalence of this condition accounts for more than $80 \%$ of oral cancers ${ }^{[10]}$. Research constantly center on the relationship between oral microorganisms and oral squamous carcinoma.

\section{Characteristics of oral microflora in oral squamous carcinoma}

Characteristics of microflora in oral squamous carcinoma tissues Aerobic bacteria dominate biomembrane on surface of healthy oral mucosa. Biomembrane on tumor surface of oral squamous carcinoma contains more aerobic and anaerobic bacteria than other organisms ${ }^{[11,12]}$. Compared with healthy mucosa, pathological changes in tumor tissues lead to changes in bacterial colonization, and microbial communities also change correspondingly. Tumor necrosis tissues provide adequate nutrients for growth and metabolism of microorganisms. In ulcerated and stealthy lacunae, oxidation-reduction potential is reduced, favoring growth of anaerobic bacteria. Decreased mucosal secretory function and rough surface of lesions affect cleaning effect of saliva. These factors also create favorable conditions for colonization and growth of anaerobic bacteria ${ }^{[13]}$. Hooper et al. ${ }^{[14,15]}$ separated living bacteria from surface and depth of oral squamous carcinoma. Their results indicated that microbial environments favor bacterial colonization and survival.

Compared with healthy mucosa environment, type and quantity of bacteria residing in squamous cell carcinoma tissues change significantly. Pushalkar et al. ${ }^{[16]}$ clinically analyzed diversity of bacteria in squamous carcinoma specimen by using $16 \mathrm{~S}$ rRNA, denaturing gradient gel electrophoresis, and cloning sequencing. Their study results showed that strains highly correlated with tumor tissue include Peptostreptococcus stomatis, Streptococcus salivarius, Streptococcus gordonii, Gemella haemolysans, Gemella morbillorum, Johnsonella ignava, and Streptococcus suis type I. Numerous experiments confirmed colonization of oral Streptococci in squamous cell carcinoma ${ }^{[17,18]}$. Populations of anaerobic bacteria, including Prevotella, Veillonella, Porphyromonas, and Capnocytophaga, also increased significantly ${ }^{[18]}$. Krogh et al. ${ }^{[12]}$ noted significantly increased amounts of Veillonella, Cyperenal, Porphyromonas, Actinomyces, Clostridium, Haemophilus, Enterobacter, and Streptococcus on surface of squamous cell carcinoma tissues. Simultaneously, some rare bacteria, such as Plantibacter flavus, Clavibacter michiganensis, and Thermus, exist in deep tissues of oral squamous carcinoma ${ }^{[14,15]}$.

In these colonized bacteria, Streptococcus anginosus is particularly noteworthy. S. anginosus exists only in dental plaques and cannot be detected in saliva. Sasaki et al. ${ }^{[19]}$ discovered high incidence of bacterial infection in patients with oral squamous cell carcinoma, and patients with $S$. anginosus detected in canceration position were also diagnosed with oral squamous cell carcinoma, which showed the same genotype of $S$. anginosus in squamous cell carcinoma region similar to that in dental plaques.

To date, squamous cell carcinoma tissues contain specific types and number of colonized bacteria. However, research on mechanism and clinical application are still lacking. Further studies should explore the role of microbial 
colonization in occurrence and development of tumors and treatment effects of controlling microbial infection on tumors to provide positive results for prevention and treatment of tumors.

\section{Characteristics of microbial community of saliva} Changes in microbial communities of saliva in oral squamous cell carcinoma patients bear significance in tumor diagnosis. Mager et al. ${ }^{[20]}$ revealed significantly increased Capnocytophaga gingivalis, Prevotella melaninogenica, and Streptococcus mitis in saliva of oral squamous cell carcinoma patients. These three bacteria act as diagnostic markers and can be used to diagnose approximately $80 \%$ of oral squamous cell carcinoma. These bacteria also feature potential in diagnosis of oral squamous cell carcinoma. However, verification of these findings still requires large-scale clinical research. Further research should elucidate causes of changes in salivary microorganisms in patients with squamous cell carcinoma. Such results may be related to secretion of secretory immunoglobulin A (sIgA). Research ${ }^{[13]}$ showed significantly reduced sIgA content in saliva of patients with oral cancer and oral mucosa carcinoma precancerosis and lower secretion speed than healthy individuals. This finding may be favorable conditions for stimulating oral infection. Saliva sampling is easily achieved in clinical practice. Future research should consider achieving early diagnosis of oral squamous cell carcinoma by using change characteristics of salivary microbial communities.

\section{Effects of oral microorganisms on occurrence of oral squamous cell carcinoma}

Multiple factors cause oral squamous cell carcinoma. To date, specific risk factors of this disease include smoking and drinking. Other related factors comprise poor oral hygiene, periodontitis, chronic bacterial infection, virus infection, or Candida infection ${ }^{[15]}$. Among these risk factors, oral microorganisms play an important role. Aside from infection, microorganisms are also closely related to periodontitis and oral hygiene and smoking and drinking.

\section{Relationship between oral microorganisms and smoking} and drinking Smoking and drinking represent two major risk factors for oral cancer. Ethanol and smog exhibit no carcinogenic effects. Both can be converted into strong carcinogens under the action of oral microorganisms. For example, ethanol is converted to acetaldehyde, a clear mutagenic and carcinogenic substance, which can interfere with synthesis and repair of DNA and induce sister chromosome exchange to generate gene mutations and cell damage ${ }^{[21]}$. Research showed significant dose -response relationship between frequency and time of ethanol intake and risk of oral squamous cell carcinoma. Gene polymorphism involved in alcohol-metabolizing enzymes can partially explain differences in susceptibility to cancer and may be one of the causes of alcohol metabolism ${ }^{[21]}$. Similarly, oral microorganisms can activate nitrosodiethylamine in tobacco ${ }^{[17]}$, forming carcinogenic substances. Smoking can also lead to increased concentration of acetaldehyde in saliva ${ }^{[22]}$, posing synergistic effects with drinking ${ }^{[23]}$.

Studies ${ }^{[24]}$ indicated that cysteine can interfere with acetaldehyde-related carcinogenesis and provided possible programs for controlling this risk factor. Cysteine can form thiazole carboxylic acid complex by combining with acetaldehyde, thus eliminating local carcinogenesis. When chlorhexidine is used for gargling before drinking, it can reduce microorganism content and $50 \%$ of acetaldehyde in saliva ${ }^{[21]}$.

Influence of oral microorganisms and chronic inflammation on occurrence of squamous cell carcinoma Oral microorganisms and their metabolites, including endotoxin, protease, collagenase, fibrinolysin, phospholipase, hydrogen sulfide, ammonia, and fatty acid with toxic effects on host cells, can directly induce cell mutation or indirectly change signal transduction pathway of cells to affect cell proliferation and activity of epithelial cells ${ }^{[25]}$. Chronic inflammation is the most direct characteristic of oral microbial infection. Chronic inflammation can indirectly activate host cells, generating hydrogen peroxide, oxygen-free radical, nitric oxide, malondialdehyde, 4-hydroxynonenal, matrix metalloproteinase, and other virulence factors, which cause cell DNA damage ${ }^{[26]}$. DNA damage caused by free radicals is the main pathological mechanism of cancer ${ }^{[27]}$. Changes in reactivity of cytokines also play an important role in cancer development.

Sasaki et al. ${ }^{[19]}$ observed that oral Streptococcus infection can induce synthesis of nitric oxide and cyclooxygenase-2, 
increase DNA damage, and promote occurrence of oral cancer. Some bacterial toxins can also interfere with signal transduction of cells and promote occurrence of tumors. For example, toxin of Pasteurella multocida and cytotoxic necrosis factor of Escherichia coli can activate signal transduction of Rho family, causing activation of cyclooxygenase-2, which participates in multiple stage tumor development ${ }^{[28]}$. Mitogens can activate transduction pathway of mitogenactivated protein kinase signaling and D1 cycle to increase gene mutation ratio and probability of cell metastasis and tumor development ${ }^{[29]}$.

In many cell signaling pathways, Toll-like receptor 5 (TLR5) shows relatively clear mechanism in occurrence of oral squamous cell carcinoma. Most cells of the immune system can express TLR5 pattern recognition receptors, which can identify bacterial flagellin and stimulate immune reactions. In malignant cells, TLR 5 not only promotes inflammatory responses but also stimulates invasion and migration of malignant cells and secretion of chemotactic cytokines. This receptor can provide conditions for development and spread of cancer ${ }^{[30]}$. Kauppila et al. ${ }^{[31]}$ detected expression of TLR5 in cancerous sites. Results showed that compared with adjacent healthy epithelium, TLR5 in cancer cells was expressed by basal layer squamous cells. Thus, expression rate of TLR5 significantly increased. Multivariate analysis showed that increased expression of TLR5 is significantly correlated to cancer incidence and cancer-related mortality.

\section{Relationship between oral microorganism in HIV-infected patients and tumors}

\section{HIV-associated tumors and non-HIV-associated tumors}

HIV infection was a significant research topic for many years. To date, HIV-positive patients are at high risk for cancer. HIV-associated tumors include non-Hodgkin's lymphoma, Kaposi's sarcoma, and invasive cervical cancer ${ }^{[32]}$. NonHodgkin's lymphoma and Kaposi's sarcoma may occur in the oral cavity. In recent years, with widespread use of highly active antiretroviral therapies, life of HIV-positive patients is prolonged. Prevalence increases for non-HIV-associated tumors, including oral squamous cell carcinoma.

In New York, non-HIV-associated tumors cause non-
HIV-related death in HIV-infected individuals. This proportion reaches as high as $21 \%$. Some studies ${ }^{[34]}$ showed that incidence of head and neck cancer in people with HIV infection is two to three times higher than that of the general population. Compared with HIV-negative population, average age of oral cancer patients among HIV-positive population is young, but survival rate is low [35]. Thus, studies should focus on HIV-infected patients suffering from non-HIV-associated tumors.

Occurrence of non-HIV-associated tumors may be related to the virus itself and its immune deficiency. HIV can directly or indirectly induce malignant tumors and directly affect cell proliferation and growth and induce integration of genome and activate oncogenes; this virus can also inhibit expression of some cytokines. These resulting factors can increase risk of cancer. HIV infection can activate chronic B cell and inhibit host immune system, leading to increased risk of cancer ${ }^{[36]}$. Immune deficiency can lead to increased risk of multiple oncogenic viral infections ${ }^{[37]}$, including combined infection of HIV and human papilloma virus (HPV)16 and HPV18. HPV infection can further increase risk of HIV-infected patients suffering from tumors.

Effects of oral microorganisms on tumor occurrence of HIVpositive patients

To date, the relationship between oral microorganisms and tumorigenesis in HIV-positive patients remains unclear. However, compared with normal individuals, HIV-positive patients present specific oral microbial communities. Microbial specificity and high incidence of cancer may exhibit some correlation.

Oral Candida albicans Oral Candida albicans is one of oral resident bacteria. Candida infection is rare in healthy population but the most common oral fungal disease in HIV-infected patients ${ }^{[38]}$. Research ${ }^{[39]}$ indicated association of Candida albicans infection of HIV patients with oral precancerous lesions. To date, oral Candida albicans infection is regarded an independent risk factor for HIV patients suffering from cancer ${ }^{[39]}$. Under HIV infection, bacteria are transformed from oral commensal bacteria to pathogenic bacteria. With phenotypic modifications and enhanced adhesion and invasion ${ }^{[40]}$, these changes may cause cancer. Although carcinogenic mechanism of 
Candida albicans requires further studies, considerable attention is paid to its effects in terms of universality of $\mathrm{HIV}$-positive infection in HIV-associated and non-HIVassociated tumors.

Microbial community of periodontal tissues Rare and severe periodontal infections, such as linear erythematous gingivitis and necrotizing ulcerative periodontitis, occur in patients with HIV infection. Subgingival microbial composition presents specificity. Paster et al. ${ }^{[41]}$ noted that subgingival microorganisms of necrotizing ulcerative periodontitis of HIV-infected patients is very similar to that of non-HIV-infected individuals. However, no typical periodontal pathogens, such as Porphyromonas gingivalis, exists. Aas et al. ${ }^{[42]}$ proved lower than normal levels of typical periodontal pathogens, including Treponema denticola, Porphyromonas gingivalis, and Tannerella forsythia, in subgingival microbial community of HIV-positive patients. Gamella, Bacteroides, Streptococcus, and Veillonella mainly comprised these communities. Moore et al. ${ }^{[43]}$ also discovered higher numbers of Leptospira and mycoplasma in subgingival flora of HIV-positive patients than those of HIV-negative individuals. These studies showed that subgingival flora in HIV-positive patients with specificity may harbor unknown specific microorganisms or microbial species that were previously thought to be incapable of causing periodontal infection. Specificity associated with high incidence of tumor requires further elucidation, but this phenomenon already caused widespread concern.

Krüger et al. ${ }^{[44]}$ reported four cases of oral squamous cell carcinoma accompanied by chronic periodontitis with HIV infection. In addition to a possible pathogenic factor, which is chronic periodontitis in one case, no other carcinogenic factors were identified. Chronic periodontitis is associated with oral squamous cell carcinoma. Tezal et al. ${ }^{[45]}$ observed that clinical attachment loss of HIV patient is associated with oral tumor and precancerous lesions. Periodontal disease may be a risk factor for oral cancer. A subsequent study ${ }^{[46]}$ proved that chronic periodontitis is a risk factor for HIV patient suffering from cancer. Immunological studies showed significantly decreased $\mathrm{T}$ cells in gingival tissues of HIV-positive patients ${ }^{[47]}$. Modifications were also noted in local immune responses, such as increased inflammatory mediators and polymorphonuclear leukocyte accumulation.
Changes in these immune factors are also risk factors for tumor development. Further research are required to identify the relationship between risk factors and tumor development.

In summary, oral microbial communities are closely related to occurrence and development of oral tumors. Studies on their relationship will provide a new direction and theoretical basis for early warning and biological treatment of tumor.

\section{Declarations}

\section{Acknowledgements}

No.

\section{Competing interests}

The author declares that he has no competing interest.

\section{Authors' contributions}

SJ Ma made the literature analysis and wrote, discussed and revised the manuscript of this review.

\section{References}

1 Aas JA, Paster BJ, Stokes LN, et al. Defi ning the normal bacterial flora of the oral cavity. J Clin Microbiol, 2005,43(11):5721-5732.

2 Allavena P, Garlanda C, Borrello MG, et al. Pathways connecting inflammation and cancer. Curr Opin Genet Dev, 2008, 18(1):3-10.

3 Warren JR, Marshall B. Unidentified curved bacilli on gastric epithelium in active chronic gastritis. Lancet, 1983, 1(8336):1273-1275.

4 Majmudar P, Shah SM, Dhunjibhoy KR, et al. Isolation of Helicobacter pylori from dental plaques in healthy volunteers. Indian J Gastroenterol, 1990, 9(4):271-272.

5 Song Q Lange T, Spahr A, et al. Characteristic distribution pattern of Helicobacter pylori in dental plaque and saliva detected with nested PCR. J Med Microbiol, 2000, 49(4): 349-353.

6 Miyabayashi H, Furihata K, Shimizu T, et al. Infl uence of oral Helicobacter pylori on the success of eradication therapy against gastric Helicobacter pylori. Helicobacter, 2000, 5(1):30-37.

7 Marshall BJ, Warren JR. Unidentifi ed curved bacilli in the stomach of patients with gastritis and peptic ulceration. Lancet, 1984, 1(8390):1311-1315.

8 Chiba T, Marusawa $\mathrm{H}$, Seno $\mathrm{H}$, et al. Mechanism for gastric cancer development by Helicobacter pylori infection. J Gastroenterol Hepatol, 2008, 23(8Pt 1):1175-1181. 
9 Ferreira AC, Isomoto H, Moriyama M, et al. Helicobacter and gastric malignancies. Helicobacter, 2008, 13(Suppl 1):28-34.

10 Kujan O, Glenny AM, Duxbury J, et al. Evaluation of screening strategies for improving oral cancer mortality: a Cochrane systematic review. J Dent Educ, 2005, 69(2):255- 265.

11 Nagy KN, Sonkodi I, Szöke I, et al. The microfl ora associated with human oral carcinomas. Oral Oncol, 1998, 34(4):304-308.

12 Krogh P, Hald B, Holmstrup P. Possible mycological etiology of oral mucosal cancer: catalytic potential of infecting Candida albicans and other yeasts in production of N-nitro sobenzylmethylamine. Carcinogenesis, 1987, 8(10):1543- 1548.

13 Lu X. Research progress of the relationship between oral squamous carcinoma and change in oral flora. Foreign Medical Stomatology, 2000, 32(5):265-267.

14 Hooper SJ, Crean SJ, Lewis MA, et al. Viable bacteria present within oral squamous cell carcinoma tissue. J Clin Microbiol, 2006, 44(5):1719-1725.

15 Hooper SJ, Crean SJ, Fardy MJ, et al. A molecular analysis of the bacteria present within oral squamous cell carcinoma. J Med Microbiol, 2007, 56(Pt 12):1651-1659.

16 Pushalkar S, Ji X, Li Y, et al. Comparison of oral microbiota in tumor and non-tumor tissues of patients with oral squamous cell carcinoma. BMC Microbiol, 2012, 12:144.

17 Yang L, Ganly I, Morris L, et al. Relevance of microbiome to cigarette smoking and oral cancer. J Dent Res, 2011, 90:120.

18 Sakamoto $\mathrm{H}, \mathrm{Naito} \mathrm{H}$, Ohta $\mathrm{Y}$, et al. Isolation of bacteria from cervical lymph nodes in patients with oral cancer. Arch Oral Biol, 1999, 44(10):789-793.

19 Sasaki M, Yamaura C, Ohara-Nemoto Y, et al. Streptococcus anginosus infection in oral cancer and its infection route. Oral Dis, 2005, 11(3):151-156.

20 Mager DL, Haffajee AD, Devlin PM, et al. The salivary microbiota as a diagnostic indicator of oral cancer: a descriptive, non-randomized study of cancer-free and oral squamous cell carcinoma subjects. J Transl Med, 2005, 3:27.

21 Reidy J, McHugh E, Stassen LF. A review of the relationship between alcohol and oral cancer. Surgeon, 2011, 9 (5):278-283.

22 Morse DE, Psoter WJ, Cleveland D, et al. Smoking and drinking in relation to oral cancer and oral epithelial dysplasia. Cancer Causes Control, 2007, 18(9):919-929. Salaspuro V, Salaspuro M. Synergistic effect of alcohol drinking and smoking on in vivo acetaldehyde concentration in saliva. Int J Cancer, 2004, 111(4):480-483.

24 Salaspuro V. Pharmacological treatments and strategies for reducing oral and intestinal acetaldehyde. Novartis Found Symp, 2007, 285:145-153.

25 Lax AJ. Opinion: bacterial toxins and cancer-a case to answer. Nat Rev Microbiol, 2005, 3(4):343-349.
26 Peek RM Jr, Mohla S, DuBois RN. Inflammation in the genesis and perpetuation of cancer: summary and recommendations from a national cancer institute-sponsored meeting. Cancer Res, 2005, 65(19):8583-8586

27 Pitot HC, Dragan YP. Facts and theories concerning the mechanisms of carcinogenesis. FASEB J, 1991, 5(9):2280- 2286.

28 Lax AJ, Thomas W. How bacteria could cause cancer: one step at a time. Trends Microbiol, 2002, 10(6):293-299.

29 Shah AA, Mayank M, Mulla AF. Evolving role of bacteria in oral cancer. Univer Res J Dent, 2012, 2(3):103.

30 Park JH, Yoon HE, Kim DJ, et al. Toll-like receptor 5 activation promotes migration and invasion of salivary gland adenocarcinoma. J Oral Pathol Med, 2011, 40(2):187-193.

31 Kauppila JH, Mattila AE, Karttunen TJ, et al.Toll-like receptor 5 and the emerging role of bacteria in carcinogenesis. Oncoimmunology, 2013, 2(4): e23620.

32 Ancelle-Park R. Expanded European AIDS case definition. Lancet, 1993, 341(8842):441.

33 Sackoff JE, Hanna DB, Pfeiffer MR, et al. Causes of death among persons with AIDS in the era of highly active antiretroviral therapy: New York City. Ann Intern Med, 2006, 145(6):397-406.

34 Cinti SK, Gandhi T, Riddell J 4th. Non-AIDS-defining cancers: should antiretroviral therapy be initiated earlier. AIDS Read, 2008, 18(1):18-20, 26-32.

35 Singh B, Balwally AN, Shaha AR, et al. Upper aerodigestive tract squamous cell carcinoma. The human immunode ficiency virus connection. Arch Otolaryngol Head Neck Surg, 1996, 122(6):639-643.

36 Herida M, Mary-Krause M, Kaphan R, et al. Incidence of non-AIDSdefining cancers before and during the highly active antiretroviral therapy era in a cohort of human immunodeficiency virus-infected patients. J Clin Oncol, 2003, 21(18):3447-3453.

37 Palefsky J. Biology of HPV in HIV infection. Adv Dent Res, 2006, 19(1):99-105.

38 Tindall B, Carr A, Cooper DA. Primary HIV infection: clinical, immunologic, and serologic aspects $[\mathrm{M}] / /$ Sande MA, Volberding PA. The medical management of AIDS. 4th ed. Philadelphia: WB Saunders, 1995:105-129.

39 Cawson RA. Leukoplakia and oral cancer. Proc R Soc Med, 1969, 62(6):610-615.

40 Agabian N, Miyasaki SH, Kohler G, et al. Candidiasis and HIV infection: virulence as an adaptive response $[M] / /$ Greenspan JS, Greenspan D. Oral manifestations of HIV infections. Chicago: Quintessence, 1995:85-92.

41 Paster BJ, Russell MK, Alpagot T, et al. Bacterial diversity in necrotizing ulcerative periodontitis in HIV-positive subjects. Ann Periodontol, 2002, 
$7(1): 8-16$

42 Aas JA, Barbuto SM, Alpagot T, et al. Subgingival plaque microbiota in HIV positive patients. J Clin Periodontol, 2007, 34(3):189-195.

43 Moore LV, Moore WE, Riley C, et al. Periodontal microflora of HIV positive subjects with gingivitis or adult periodontitis. J Periodontol, 1993, 64(1):48-56.

44 Krüger M, Hansen T, Kasaj A, et al. The correlation between chronic periodontitis and oral cancer. Case Rep Dent, 2013, 2013:262410.
45 Tezal M, Grossi SG, Genco RJ. Is periodontitis associated with oral neoplasms. J Periodontol, 2005, 76(3):406-410.

46 Tezal M, Sullivan MA, Reid ME, et al. Chronic periodontitis and the risk of tongue cancer. Arch Otolaryngol Head Neck Surg, 2007, 133(5):450-454.

47 Glick M, Muzyka BC, Salkin LM, et al. Necrotizing ulcerative periodontitis: a marker for immune deterioration and a predictor for the diagnosis of AIDS. J Periodontol, 1994, 65(5):393-397. 